\title{
Constructions identitaires en mouvement dans le récit de voyage Önskeresan de Göran Schildt
}

Harri Veivo

Université de Caen Normandie

À la fin des années I940, de nombreuses villes européennes - comme Cologne, Rotterdam, Le Havre - sont en ruines et plusieurs milliers de personnes vivent dans des camps de transit dans l'attente - ou la crainte - d'un retour au pays. L'économie peine à redémarrer, les aliments de base restent rationnés, les pays de l'Europe centrale et de l'Est sont en train de basculer dans le totalitarisme et la culture cosmopolite et multi-ethnique de ces pays s'est noyée dans la terreur de la guerre et de la Shoah. La liste des calamités est longue, mais il nous faut souligner ici que les ravages de la guerre ont également contribué à une possibilité de changement, qui devient indispensable. «La guerre a tout changé, écrit l'historien Tony Judt, un retour à l'état d'avant I939 était hors de question presque partout ${ }^{\mathrm{I}}$ " (Judt, 2010 : 63). C'est dans ce contexte de contrastes, de ruptures et de déchirures que l'écrivain et historien d'art Göran Schildt (I9I7-2009) entame un voyage en bateau à voile de Stockholm à Rapallo via le Danemark, l'Angleterre et la France. Il a d'abord publié le récit de son voyage sous forme d'articles dans le quotidien suédois Svenska Dagbladet, puis en livre Önskeresan [ Voyage de rêve »] en I948. Succès de librairie, il est bientôt traduit en six langues; les ventes permettent à Schildt de devenir écrivain professionnel et de continuer ses voyages en Mer d'Egée, en Mer Noire, sur le Nil et ailleurs. Pour de nombreux lecteurs, Önskeresan a sans doute été un contrepoids à l'isolement de l'après-guerre ainsi qu'un important facteur dans la reconstitution de l'image d'une Europe divisée après les horreurs de la guerre, tout en offrant en même temps le modèle d'un homme libre et authentique

How to cite this book chapter:

Veivo, H. 2015. Constructions identitaires en mouvement dans le récit de voyage Önskeresan de Göran Schildt. In: Cedergren, M. et Briens, S. (eds.) Médiations interculturelles entre la France et la Suède. Trajectoires et circulations de 1945 à nos jours. Pp. I 8-I29. Stockholm: Stockholm University Press. DOI: http://dx.doi.org/I0.I6993/ bad.j. License: CC-BY 
(voir Zilliacus, 2000 : 2I 5). Pour Schildt, cela représentait plus encore. Grièvement blessé à la guerre, il s'était promis de faire ce voyage " de rêve » s'il parvenait à survivre. Ce texte devient alors un acte de réaffirmation et de reconstruction de l'identité personnelle, mais en même temps un acte de fidélité et de reprise de contacts et appartenances plus anciennes avec la France qui a joué un rôle prépondérant dans la vie de l'auteur avant la guerre. Cet acte situe Schildt dans le croisement de plusieurs cultures, dont il sera le passeur et le médiateur. Écrivain svécophone finlandais, installé en Suède après la guerre et parfaitement intégré à la vie culturelle de Stockholm, Schildt construit son récit sur l'Europe par rapport à la Finlande, la Suède et l'espace nordique. En même temps, il développe une identité cosmopolite en esprit et dans la vie pratique : passionné par la culture européenne, il est prêt à partager l'espace exigu de son bateau avec des amis de nationalités différentes ou à provoquer des rencontres inattendues avec des inconnus dont il croise le chemin. Dans le récit qu'il livre de son voyage, ces deux dimensions entrent dans un jeu réciproque, les péripéties du voyage et les rencontres déplaçant les repères identitaires et bousculant les conceptions sur les pays et les cultures que l'écrivain développe dans un travail de réflexion constant.

Dans cet article, je vais en premier temps situer Göran Schildt dans le contexte de son temps, en me focalisant notamment sur sa position particulière entre générations et classes sociales et sur son internationalisme et sa francophilie affirmés. Ces facteurs ont conditionné la construction identitaire de l'écrivain, constituant un fil qui relie les expériences de jeunesse de l'avant-guerre avec les essais et les récits de voyage de l'après-guerre. Je vais ensuite analyser Önskeresan avec une attention particulière sur la façon dont la perception et la conception que l'écrivain a des pays, cultures et nations évoluent sous l'influence de la mobilité ${ }^{2}$. La fin de l'article sera consacrée à une discussion sur le cosmopolitisme de Schildt, vu notamment par rapport aux débats contemporains sur la potentialité critique de sujets transnationaux et les conditions de partage des valeurs, idées et émotions au-delà des frontières nationales. Ces thèmes sont redevenus d'actualité dans le contexte de la globalisation des rapports politiques, économiques et culturels ainsi que par la mobilité accrue de populations à l'échelle mondiale; il sera intéressant de voir comment le cas Schildt, sujet mobile entre sphères cultures différentes et en déplacement dans un paysage de ruines et de reconstruction, peut contribuer à cette réflexion. 


\section{Göran Schildt entre classes et générations}

Schildt est revenu sur son enfance et l'histoire de sa famille à de nombreuses reprises. Pour notre thème, ses mémoires Lånade vingar (I995) et Tvivlets gåva (2000) constituent des sources de première importance. Dans ces livres, Schildt raconte notamment l'histoire de son père, l'écrivain Runar Schildt ( I888-I925), et les conséquences que son suicide a eues sur sa jeunesse. La famille Schildt, d'origine allemande, a acquis ses titres de noblesse en Suède en I 642 puis en Finlande en I 8 I 8 . La branche finlandaise compte dans ses rangs des médecins, des grands propriétaires fermiers, mais aussi des hommes politiques acquis à la cause de l'éveil national finlandais. Le père de Runar Schildt, souffrant de troubles psychiques et incapable de faire carrière, s'était marié avec une femme d'origine modeste, fille d'un cordonnier. Après la mort précoce de son père, Runar Schildt grandit dans une situation économiquement difficile et socialement située entre les classes avec à la fois un pied dans la noblesse et l'autre dans la classe ouvrière. Selon Göran Schildt, cette position a fait de son père un étranger au sein de sa propre société : "Il est devenu un observateur étranger qui regardait avec une certaine jalousie l'assurance et la solidarité des "forts", mais ne sentait d'affinité qu'avec les "faibles", les vaincus et les exclus » (Schildt, I 995 : 4I). La littérature est devenue dans ce contexte un moyen de réussite sociale pour Runar Schildt ; lorsqu'il a senti sa créativité s'épuiser, il a choisi le suicide, craignant de devenir " un homme complètement inutile et insignifiant " (lettre d'adieu de Runar Schildt, citée dans Schildt, I995 : 34). Selon l'interprétation de Göran Schildt, son père a fait sa carrière littéraire trop tôt, dans une situation historique où les écrivains étaient toujours dépendants du soutien d'un public conservateur et, par voie de conséquence, de leur approbation morale. Ce n'est que la génération des modernistes des années I920 - représentée par Hagar Olsson, Elmer Diktonius, Rabbe Enckell, Gunnar Björling par exemple - qui a pu émanciper la littérature de cette position.

Schildt affirme que l'héritage de son père - et notamment son œuvre littéraire - a " exercé une influence sur [lui] dans le sens où il [1]'a poussé à continuer les questionnements de [son] père, mais [1]'a aussi incité à le contredire »(Schildt, 2000: 26). Des éléments similaires se retrouvent en effet dans le récit que Göran Schildt offre de sa propre jeunesse, et le thème fondateur de l'œuvre du père, «le problème de savoir si un homme peut et doit rester un observateur extérieur par rapport à la réalité » (Schildt, 2000: 27), joue également un rôle central dans l'œuvre du fils. 
Après la mort du père, la famille a des revenus sûrs, mais relativement modestes. Schildt grandit pourtant protégé par les amis de son père, dans un contact proche avec la haute bourgeoisie d'Helsingfors. Il arrive à la majorité dans les années I930, dans un contexte culturel où la percée du modernisme recule face à l'endurcissement du climat idéologique en Europe et en Finlande. Après avoir obtenu son baccalauréat, Göran Schildt reçoit un héritage qui lui permet de passer l'année I934-5 en France où il visite d'abord le Midi et s'inscrit ensuite à la Sorbonne. À Paris, il habite chez Mme de Coppet, la veuve du premier ambassadeur de la France en Finlande. Le séjour en France est pour Göran Schildt un véritable acte de construction identitaire. "Ce qui m'attirait, écrit-il dans Lånade vingar, était la liberté de l'esprit, les traditions humanistes et l'aventure créative, caractéristiques que j'associais spontanément à la France »(Schildt, I995 : 60). Grâce à ces valeurs que Schild attache à la France, le pays représentait un contre-modèle à l'Allemagne et l'Angleterre. L'Allemagne, une destination traditionnelle pour les étudiants finlandais et une source d'influence importante pour les modernistes des années 20, était jugé trop discipliné et collectif par le jeune Schildt, et l'Angleterre, le choix de son frère Christoffer, trop conventionnel et matérialiste. Un voyage en Italie en I937 avec Georg Henrik von Wright, le futur professeur de philosophie à Oxford et à Helsingfors, permet d'approfondir cette recherche des valeurs humanistes et met en place le fondement d'un dispositif de comparaison des pays et des cultures, qui s'attache à l'opposition du « Nord » et du "Sud» et qui se retrouve dans Önskeresan.

Après son séjour parisien, Göran Schild étudie à l'Université d'Helsingfors, où il suit notamment les cours d'Yrjö Hirn, dont la longue carrière avait déjà influencé la génération de son père et celle des modernistes des années 20. Hirn défendait une conception kantienne mettant en valeur la nature désintéressée de la contemplation esthétique et l'autonomie de l'art et de la littérature (voir par exemple Zilliacus, 2000 : 206-208). En opposition à Hirn, Schildt a alors forgé sa propre conception, insistant sur la valeur heuristique de l'art dans l'existence et sa capacité à fournir "du matériel pour une vision du monde pratique, des clés pour interpréter l'existence et une échelle de valeurs à suivre »(Schildt, I995: 46). D'autre part, il raconte avoir souffert d'une conscience trop aigue, d'un réflexe d'observation inévitable face à l'existence, se rendant ainsi étranger à la vie pratique et active. Si ces propos rappellent l'œuvre et le destin tragique de son père, c'est la culture française, et plus spécifiquement Paul Cézanne et André Gide, 
sujets de deux essais écrits pendant la guerre, qui a aidé le jeune Schildt à réfléchir sur ce handicap intellectuel. Dans la vie de Cézanne, il aperçoit un clivage entre le moi esthétique de l'artiste et son moi quotidien. Ce clivage produit ce que Schildt appelle «le dilemme » ou «cercle vicieux » romantique et qui frappe la vie quotidienne de négativité (Schildt, I946a : 38-39, 76). Il aperçoit le même phénomène dans la vie et l'œuvre de Gide, mais alors que Cézanne cherchait à échapper au dilemme par une réinvention du classicisme, Gide est prêt à poursuivre "la spontanéité dans l'expérience de réalité », même au prix d'actes que la société considère comme immoraux (Schildt, I946b : 54). Écrivain courageux montrant la voie de sortie du dilemme personnel, Gide est devenu pour Schildt une figure salutaire et le compagnon d'un dialogue épistolaire qui s'est étendu sur plusieurs années.

Si l'on compare ces deux essais, on aperçoit que celui sur Gide, fruit d'un travail de longue haleine entamé dans les années I930 et écrit en Finlande pendant la Deuxième Guerre Mondiale, se distingue à la fois par la perspicacité des analyses et l'absence quasi-totale de références à d'autres ouvrages sur l'auteur ; il s'agit clairement d'un essai sur l'œuvre de Gide et sur les dilemmes personnels de l'auteur de l'essai. Dans ce sens, il caractérise à l'extrême le trait constitutif de la francophilie de Schildt que nous avons évoqué plus tôt dans le commentaire sur le choix entre l'Allemagne, l'Angleterre et la France : l'intérêt qu'il a porté sur la culture française est motivé par le besoin de forger son identité personnelle à travers un dialogue avec des figures tutélaires dont les préoccupations correspondent à sa situation existentielle particulière. L'étude sur Cézanne est par contre plus ancrée dans les discussions contemporaines sur l'histoire de l'art moderne. Schildt utilise à la fois sa connaissance des mouvements internationaux et du domaine finlandais, caractérisant par exemple l'expressionisme dans les arts visuels par référence au poète moderniste finlandais Elmer Diktonius. L'essai continue la tradition du cosmopolitisme comme "stratégie locale » typique à la génération des écrivains svécophones de la Finlande des années 20 : la connaissance de l'art international permet à l'auteur avant tout d'acquérir du capital culturel dans le champ national (la Finlande) et régional (la Finlande et la Suède ; voir Nyberg, 20I2). Bien qu'il s'agisse d'une œuvre commandée pour une série de monographies sur les peintres contemporains, l'essai fût accepté comme thèse de doctorat à l'université d'Helsingfors en 1947. Schildt a cependant renoncé à la carrière universitaire qui s'ouvrait à lui, préférant la liberté de l'écrivain aux contraintes du professeur, ce qui ne l'a pas empêché de devenir un spécialiste reconnu de l'œuvre d'Alvar Aalto. 


\section{Constructions mobiles du " nord " et du " sud "}

Le récit de voyage Önskeresan s'inscrit dans la continuité des essais sur Cézanne et Gide et de la réflexion artistique, intellectuelle et existentielle qui les motive. Si les deux essais tournent autour du dilemme existentiel de l'auteur, déchiré entre l'art et la vie, la contemplation et la participation, le voyage et l'acte de le configurer dans le récit lui offrent un moyen de le dépasser. Le mode de transport, le bateau à voile Daphne, joue un rôle important ici, tout comme le travail d'écriture. La voile est pour Schildt le moyen d'affirmer à la fois « la conscience dont [il] ne peut pas se libérer et la fusion avec le moment présent qu'[il] appelle le flot de la vie " (Schildt, I995 : 2 I4). En même temps, l'écriture de voyage lui offre la possibilité de réfléchir sur la culture, les arts et la tradition humaniste européenne, de mettre à profit ses connaissances encyclopédiques, et de lier ces réflexions et connaissances à la narration des événements, aux actions et rencontres qui ont lieu dans la vie pratique et concrète, et qui mettent parfois en question les conceptions théoriques et les représentations figées de l'auteur. Il faut souligner ici le rôle fondamental de la mobilité. S'il est vrai, comme l'écrit Jean-Marc Moura, que « l'appréhension d'une réalité étrangère par un écrivain [...] n'est pas directe, mais médiatisée par les représentations imaginaires du groupe ou de la société auxquels il appartient » (Moura, I998:45) et que la question de référence peut ainsi être mise en parenthèses dans l'analyse, il est important de prendre également en considération "les lignes de résistance » (Eco, I999 : 26I) que la réalité peut opposer aux représentations et qui conditionnent leurs conditions de félicité sans pour autant déterminer leurs contenus. La mobilité fait ressortir cette résistance ou friction et montre ainsi les limites des représentations imaginaires ; elle a une potentialité critique qui concerne tout aussi bien le sujet observant et écrivant que l'objet décrit dans la narration.

Le voyage de Schildt, de Stockholm à Rapallo, peut être caractérisé comme un déplacement graduel d'une aire culturelle familière et quotidienne vers un " ailleurs " qui, bien que connu grâce aux voyages de l'avant-guerre, reste un domaine inconnu, objet de découvertes et projections. Il s'agit d'un voyage qui va du " même » vers ce qui est "différent ", donc d'une traversée de frontière, mais comme ces notions sont relatives et non pas absolues, le voyage déplace la frontière et la repousse toujours plus loin. Comme l'écrit Schildt, lors du passage à Rouen, ville exotique et méridionale pour l'auteur dans sa jeunesse mais nordique et ennuyeuse pour Flaubert, « ce qui est le sud 
pour l'un peut être le nord pour un autre. Le sud est une direction, pas un lieu " (Schildt, I957:83). Cette "vérité triviale" produit dans le texte un jeu complexe de positionnements relatifs rendus dynamiques par la mobilité qui ne laisse aucun terme intact. Ce jeu repose sur des comparaisons qui établissent des similarités et des différences, mais qui permettent aussi d'apercevoir les entités mises en relation «les un[e]s à travers les autres, en termes de relations, d'interactions, de circulation » (Werner \& Zimmermann, 2003 : I 5-I6). Dans l'œuvre de Schildt, ce n'est pas uniquement le poète finlandais Diktonius qui sert à caractériser l'expressionisme français au public suédois ; d'autres éléments aussi sont susceptibles de changer de place et de signification afin d'ouvrir de nouvelles perceptions aux phénomènes qu'ils mettent en valeur.

Notons d'abord que, même si l'opposition structurante du récit est celle de « Nord » vs. «Sud », l'espace nordique n'est pas pour Schildt une entité homogène ni un donné immuable. L'arrivé au Danemark amène l'auteur à développer une réflexion sur les particularités des sociétés danoise, suédoise, norvégienne et finlandaise et leurs différences. Si le Danemark apparaît comme un pays où les distinctions entre classes sociales ont disparu, donnant lieu à un partage de valeurs fondamentales à l'échelle de toute la nation, la Suède est un pays marqué par l'incertitude et la vacuité qui découlent du déni généralisé de l'existence de classes sociales. Ces deux pays négocient donc différemment la modernisation des sociétés, alors que, en comparaison, la Norvège et la Finlande sont toujours marquées par la persistance de la tradition maritime ou de l'esprit pionnier qui leur procurent une mentalité vivante et une force morale (Schildt, I957 : 37-38). Cette série de distinctions est cependant à géométrie variable et susceptible d'être actualisée et interprétée de manière flexible. Naviguant sur le Canal de Bourgogne, l'équipée du Daphne rencontre deux Danois partis eux aussi pour un voyage en bateau vers la Méditerranée. Cet événement inattendu évoque d'abord la joie de la reconnaissance d'une identité partagée : "Plus on s'éloigne de chez soi, écrit Schildt, plus on se sent compatriote avec tous les Scandinaves » (Schildt, I957 : I6I). Ce sentiment est pourtant mis à l'épreuve par la différence entre les comportements des deux équipées, l'esprit bohème des Danois et le désordre qui règne dans leur bateau contrastant fortement avec le sérieux de Schildt et le renvoyant dans la position inconfortable d'un « propriétaire ploutocrate d'un yacht luxueux »(Schildt, I957 : I6I). D'autre part, plus tôt dans le récit, l'étape en Angleterre et l'arrivée en France - au port du Havre dévasté par les bombardements, puis à Rouen - concluent à une comparaison 
reposant sur la conception d'un espace nordique homogène. Les pays nordiques et la France sont opposés en tant que pays démocratiques et égalitaires, à l'Angleterre qui apparaît en même temps comme une société de classes mais aussi comme le pays héritier de la civilisation grecque dans sa volonté forger un " gentleman idéal » qui serait avant tout un " être social» (Schildt, I957:65). L'espace nordique est ainsi susceptible de fragmentation ou de cohérence selon la fonction qu'il occupe dans la réflexion de Schildt : espace de différences localisées dans le Nord lorsqu'il sert à caractériser et critiquer la société suédoise ; espace cohérent lorsqu'il entre en constellation avec d'autres pays et sert à rendre visible leurs similarités et différences.

Le jeu de positionnement que Schildt développe connaît donc des niveaux de granularité différents qui rendent visibles des clivages et rapprochement variables. L'opposition structurante reste celle de «nord » vs. "sud ", mais il est important de noter que la mobilité du sujet et la variabilité des niveaux de comparaison structurent cette opposition autour de trois termes et non pas uniquement deux. La différence entre la France et l'Angleterre n'apparaît pas grâce à une mise en relation de deux éléments, mais de trois ou quatre : ce sont les pays nordiques et l'héritage commun des pays européens qui fondent cette relation en fournissant les termes de comparaison et qui sont la démocratie et l'égalité dans la société contemporaine d'une part et l'héritage de la civilisation grecque ancienne de l'autre part. À la fin du récit, l'opposition fondamentale est encore une fois reconfigurée. Si la France avait jusqu'ici été - malgré son assimilation avec les pays nordiques noté supra - plutôt marquée par une altérité par rapport à la Suède, visible dans les mœurs, les comportements et les façons de parler, elle devient à la fin résolument catégorisée comme appartenant au "nord » par rapport à l'Italie. "Tout d'un coup j'ai compris, écrit-il en décrivant le moment de débarquer à San Remo, que la frontière véritable entre le sud et le nord passe entre la France et l'Italie. Toutes les faiblesses du caractère français se dévoilaient sans frémir devant ma conscience, parce qu'ils étaient les miens, parce qu'ils étaient nordiques » (Schildt, I957 : 224). L'arrivé en Italie marque pour Schildt la découverte d'une autre manière d'être qui est plus insouciante, plus humaine et en apparence plus superficielle que dans le "nord ", mais qui est en vérité le fruit d'une longue maturation millénaire qui a réussi à unir la philosophie et la vie quotidienne. Cette manière d'être rappelle la conception d'art que le jeune Schildt s'était forgée, tout comme "l'angoisse métaphysique, le gouffre béant entre l'être et le paraître, le sentiment d'insatisfaction 
qui transforme notre vie en ombre de quelque chose que nous ne pouvons jamais atteindre "(Schildt, I957 : 224) qui marquent les pays nordiques et la France est semblable au cercle vicieux de négativité qu'il a trouvé dans l'œuvre de Cézanne, Gide et son père. Le voyage vers l'ailleurs est donc un processus où la frontière toujours déplacée entre le même et l'ailleurs et la confrontation continue avec l'altérité produisent de nouvelles configurations et finissent par faire fusionner la France avec le "nord "; en même temps, le voyage est une redécouverte de soi - d'un soi forgé par la littérature et l'art.

\section{Schildt, sujet cosmopolite}

De nombreuses recherches publiées pendant les vingt dernières années ont mis en valeur les notions du cosmopolite et du cosmopolitisme dans les débats sur les identités et appartenances dans un monde postcolonial et globalisé. La multiplication des phénomènes de migration rend nécessaire le développement d'une conscience d'appartenance à une communauté supra-nationale, et de devoirs et compétences permettant d'y vivre en bonne entente (voir par exemple Appiah, 2006, Zarka, 20I4). Dans ce contexte, les sujets postcoloniaux, dont l'existence est conditionnée par les puissances hégémoniques et qui sont donc complexes dès l'origine, peuvent présenter une potentialité critique signifiante, puisque leur position liminale, entre intégration et exclusion, fait apparaître des failles dans les constructions épistémologiques et identitaires euro- ou ethnocentriques (Mignolo, 2000a et 2000 b). Schildt n'est certes pas un sujet postcolonial dans le sens habituel du mot. En tant que finlandais et svécophone, il est cependant situé à l'intersection des blocs géopolitiques en train de former dans l'Europe de l'après-guerre, et membre d'une communauté linguistique minoritaire dans son propre pays. Son déménagement en Suède est partiellement motivé par la crainte de voir la Finlande basculer dans le giron de l'Union Soviétique. D'autre part, il est un sujet suédois par sa langue et ses contacts dans la société, et donc représentant d'un pays qui a su rester neutre pendant la guerre et l'est dans le contexte de Guerre froide aussi. Cette appartenance conjointe à deux mondes offre à Schildt une position privilégiée d'observateur critique et une flexibilité dans ses appartenances identitaires ${ }^{3}$. La manière dont celles-ci sont conditionnées et soumises à médiation dans son récit appellent une analyse en ces termes et présentent un intérêt pour le débat plus général sur le cosmopolitisme.

Comme nous l'avons vu, le travail incessamment repris de réflexion sur les traits particuliers des pays, nations et régions repose pour une 
grande partie sur la conception de l'art et de l'existence que Schild s'est forgée dans sa jeunesse. De là même manière, il se définit, à son arrivée en Italie, moment clé dans le récit, comme sujet-observateur par rapport à des figures tutélaires du monde des arts et lettres qui médiatisent sa relation par rapport à l'environnement et dont Goethe et Stendhal sont les plus importants. L'amour du Sud de Goethe est pour Schild exemplaire dans le sens où l'écrivain allemand a su fusionner sa vie avec la culture du pays qu'il a découvert et qu'il a adoré, alors que pour Stendhal, tout aussi amoureux de l'Italie, la découverte du pays n'a pas abouti à une fusion heureuse, mais a plutôt ouvert une faille existentielle. Dans l'œuvre de Stendhal, l'Italie tant adorée frappe de négativité la France, dont l'écrivain n'a pourtant pas pu s'émanciper. L'enjeu est donc ici aussi la possibilité où l'échec de trouver une harmonie entre les idéaux et la réalité, entre l'art et la vie quotidienne. Si cette réflexion provient de la lecture des grands auteurs de la culture européenne, étant donc par nature philosophique et théorique, Schildt participe tout le long de son voyage à des négociations identitaires, liées d'une part à son origine, et d'autre part et à la vie quotidienne concrète et immédiate. En tant que citoyen finlandais svécophone maîtrisant le finnois, il peut se positionner comme finlandais, suédois ou scandinave, en fonction de la situation où il se trouve. Ainsi, à l'arrivé à Aalborg au Danemark, il rencontre le contrebandier quasi mythique Algot Niska, avec qui il entame une discussion en finnois avant de changer pour le suédois ; le personnage de Niska évoque à Schildt « la mentalité directe qui était à la fois internationale et typique aux villes de province et qui caractérisait la Finlande impériale de nos grands-parents » (Schildt I957 : 44). Si cette rencontre ramène à la surface une particularité de l'identité finlandaise, la période historique - positivement évaluée - passée sous l'Empire russe, dans d'autres occasions Schildt se positionne pleinement comme un sujet suédois ou nordique, se réjouissant des rencontres avec des personnes originaires de la même région. Cette flexibilité ouvre à Schildt une sphère de contacts très étendue, mais lui fait prendre conscience en même temps des limites d'appartenance marquées par d'autres facteurs que linguistiques, comme par exemple dans l'épisode de la rencontre avec les deux Danois bohèmes sur le Canal de Bourgogne.

En addition aux identités "philosophique ", " nordique » et " finlandaise ", Önskeresan évoque aussi un troisième plan où les relations de partage et d'appartenance se jouent : la vie active et concrète conditionnée par le bateau et soumise à la médiation par les activités qu'elle nécessite. L'équipage du Daphne et son capitaine sont constamment 
amenés à coopérer avec des inconnus : marins, officiers de la douane, pilotes, éclusiers, - et les innombrables curieux que leur bateau attire. Cette coopération n'efface pas les différences de classe, les préjugés ethniques ou les difficultés liées à la langue, mais elle permet de les relativiser et d'entamer des actions partagées qui visent un objectif reconnu par tous les participants de l'action. En ce sens, il s'agit d'une " cosmopolitique affective de subjectivités immanentes et incarnées » (Braidotti, Blaadgaard \& Hanafin, 20I3 : 3) qui va au-delà du cosmopolitisme philosophique fondé sur la théorie ou la connaissance abstraite. Cette dimension concrète est une composante essentielle de l'identité de Schildt, car c'est justement cet élément qui permet de renouer les connaissances accumulées dans la lecture et les appartenances qu'elles permettent - le cercle des écrivains et intellectuels, morts et vivants : Goethe, Stendhal, Cézanne, Gide - avec des expériences vécues ensemble et situées dans des lieux spécifiques et des moments précis. Dans ce sens, et ceci peut être un facteur qui explique le succès du livre, Önskeresan montre, dans le contexte difficile de l'après-guerre, une manière de vivre la culture européenne et de partager cette expérience dans l'ici et maintenant, au-delà des clivages entre les langues et les nationalités, sans être englouti par le poids des tragédies passées ni violemment projeté vers un avenir incertain. Par le récit, cette expérience aspire à la transcendance. Le cosmopolitisme de Schild est inséparable de ses deux supports qui sont le bateau à voile et l'écriture. Si le premier ancre l'identité dans des actions, lieux et moments tout en rendant possible la mobilité, le deuxième tend à l'abstraction et à la mise en forme artistique; tous les deux sont en même temps des vecteurs de partage.

\section{Notes}

I. Citations de sources suédoises et anglaises traduites par l'auteur de l'article.

2. Le récit de voyage existe sous trois formats : les articles originaux, Önskeresan et le fac-similé du livre de bord de Daphne Loggbok Stockholm - Rapallo publié en I966. Cette dernière est représentée par Schildt ironiquement comme une contribution à la mode de littérature expérimentale, mais aussi plus sérieusement comme une version alternative du récit complémentaire à celui qu'on peut trouver dans Önskeresan et qui est, pour l'auteur, plus réelle grâce à son travail de mise forme (Schildt, I966: 5-8). Ces réflexions appellent une analyse sérieuse et un débat théorique auxquels je dois pourtant renoncer ici, faute d'espace suffisant.

3. Pour la position de Schildt dans le débat concernant l'avenir de la culture et de l'identité svécophones de la Finlande, voir Schildt (20I4). 


\section{Bibliographie}

Appiah, K. (2006), Cosmopolitanism. Ethics in a World of Strangers. New York \& London : Norton.

Braidotti, R., Blaadgaard, B. \& Hanafin, P. (2013), "Introduction ", in Braidotti, R., Hanafin, P. \& Blaadgaard, B. (éd.), After Cosmopolitanism. Abingdon : Routledge : I-7.

Eco, U. (I999), Kant et l'ornithorynque (trad. par Gayrard J.). Paris : Grasset. Judt, T. (2010), Postwar. A History of Europe since 1945. London : Vintage.

Mignolo, W. (2000a), Local Histories / Global Designs. Coloniality, Subaltern Knowledge and Border Thinking. Princeton : Princeton University Press.

- (2000b), "The Many Faces of Cosmo-Polis: Border Thinking and Critical Cosmopolitism ", Public Culture 3 (vol. I2) : 72 I-748.

Moura, J.-M. (1998), L'Europe littéraire et l'ailleurs. Paris : Presses Universitaires de France.

Nygård, S. (2012), "The National and the International in Ultra (I922) and Quosego (r928) » in den Berg (van), H., Hautamäki, I. \& al. (éd.), A Cultural History of the Avant-Garde in the Nordic Countries I900-I925. Amsterdam : Rodopi : 337-350.

Schildt, G. (I946a), Cézanne. Stockholm : Wahlström \& Widstrand.

— (1946b), Gide och människan. Helsingfors : Söderström.

— (I957) [1949], Önskeresan. Stockholm : Bonniers.

— (I966), Loggbok Stockholm - Rapallo. Helsingfors : Söderström.

- (1995), Lånade vingar. Ungdomsminnen. Helsingfors : Söderström.

- (2000), Tvivlets gåva eller Förfligna tankar om Guds död och en försvunnen far. Helsingfors : Söderström.

— (2014) [1959], "Finlandssvenskarna ». [En ligne] (dernière lecture le I 5 janvier 20I4). http://www.svd.se/kultur/understrecket/finlandssvenskarna_ 2659467.svd

Werner, M. \& Zimmermann, B. (2003), «Penser l'histoire croisée : entre empirie et réfléxivité ", Annales. Histoire, Sciences Sociales I : 7-36.

Zarka, Y. Ch. (20I4), Refonder le cosmopolitisme. Paris : Presses Universitaires de France.

Zilliacus, C. (2000), «Forskare, essäister och färdmän », in Zilliacus, C. (éd.), Finlands svenska litteraturhistoria. Andra delen : I900-talet. Helsingfors/ Stockholm : Svenska litteratursällskapet i Finland/Atlantis : 206-2 I 5. 